\title{
LE PROBLEME DE LA MULTIVALENCE DANS LE TRAVAIL CONTINU
}

\author{
Jacques-André Bartoli - Raymond Trémolières \\ I.A.E. - Université de Droit, d'Economie et \\ des Sciences d'Aix-Marseille \\ 29, Av. R. Schuman - 13617 AIX-EN-PROVENCE - FRANCE
}

\begin{abstract}
PRESENTATION
L'étude généralise un précédent travail consacré à l'automatisation des plannings de roulements de quarts. Alors que la première étude concernait le travail posté en monovalence, nous abordons ici le problème de la multivalence, situation plus générale dans laquelle un ou plusieurs opérateurs peuvent travailler en alternance sur plusieurs postes. Cette hypothèse permet d'étudier le cas où l'on affecte un nombre fractionnaire d'ouvriers sur un même poste. De tels problèmes se posent assez souvent dans le travail continu où l'on cherche à déterminer, compte-tenu d'un certain taux d'absentéisme, le nombre minimum d'ouvriers nécessaire pour assurer un travail donné. La constitution des plannings en roulements de quarts étant chose plus que fastidieuse, sinon impossible à échelle humaine, nous donnons ici les moyens d'obtenir des plannings cycliques en multivalence de façon automatique.
\end{abstract}

\section{INTRODUCTION}

Cet article fait suite à une première étude (cf. (1)) consacrêe à la génération automatique de cycles de roulements de quarts en monovalence. Pour familiariser le lecteur avec le problème, nous donnons ci-après un planning de roulements de quarts à la Raffinerie de Reichstett.

Cette étude a été réalisée pour la Compagnie Rhénane de Raffinage de Reichstett (Alsace) 


\begin{tabular}{|c|c|c|c|c|c|}
\hline Semaine & 1 & 2 & 3 & 4 & 5 \\
\hline Opérateurs & $\mathrm{LM} \mathrm{MV} \cdot$ & I M M JV.. & I M M J V... & I M M J V.. & LM M J . . \\
\hline $1 \longrightarrow$ & N N N N N N N & -- S S S S S & $S S-M M M M$ & M M M - - - & $J J J J \ldots$ \\
\hline $2 \longrightarrow$ & $J J J J-\cdots$ & $N \mathrm{~N} N \mathrm{~N} N \mathrm{~N}$ & -- s s S S S & $S S-M M M M$ & M M M - - - \\
\hline $3 \longrightarrow$ & M M M - - - & $\mathrm{J} J \mathrm{~J} J$ & $N N N N N N$ & -- s s S S S & $S S-M M M M$ \\
\hline $4 \longrightarrow$ & $S S-M M M M$ & IM M M - - - & $J \mathrm{~J} J \mathrm{~J}-\cdots$ & $\mathrm{N} \mathrm{N} \mathrm{N} \mathrm{N} \mathrm{N} \mathrm{N} \mathrm{N}$ & $--S S S S S$ \\
\hline $5-$ & - - s s s s s & $S S-M M M M$ & MMM --- & $\mathrm{J} J \mathrm{~J} J$ & $N N N N N N$ \\
\hline
\end{tabular}

TABLEAU I : Roulements de quarts standard de $40 \mathrm{~h}$. (Reichstett), cycle de 5 semaines

Chaque ligne de ce tableau définit le planning de travail d'un ouvrier. Les colonnes correspondent aux jours de la semaine. Le planning se répète identiquement à lui-même de 5 semaines en 5 semaines. On remarquera (et nous prendrons aeci comme hypothèse) que les ouvriers ont tous, à un décalage près dans le temps, les mêmes plannings.

Dans le tableau ainsi que dars le texte, les symboles $N, S$, M, J définissent les "quarts" de travail dont la durée est généralement de $8 \mathrm{~h}$.

$$
\begin{aligned}
& \mathrm{N}=\text { nuit, gênéralement de } 21 \mathrm{~h} \text { à } 5 \mathrm{~h} \\
& \mathrm{~S}=\text { soir, généralement de } 13 \mathrm{~h} \text { à } 21 \mathrm{~h} \\
& \mathrm{M}=\text { matin, générâlement de } 5 \mathrm{~h} \text { à } 13 \mathrm{~h} \\
& \mathrm{~J}=\text { "journée normale", généralement de } 8 \text { heures. }
\end{aligned}
$$

Les symboles "-" ou "." marqueront une journée de repos.

Nous appellerons "tour" une suite ininterrompue d'un même quart, et "cycle" le planning d'un opérateur, qui se répète identique à lui-même.

Ainsi, dans le tableau 1, l'ouvrier 1 commence par un tour de 7 nuits et son cycle est de 5 semaines.

Dans ce tableau, on peut vérifier que chaque "jour" ou, plus exactement que dans chaque colonne, il $\mathrm{y}$ a toujours un $N$, un $S$, et un $M$. Autrement dit, $24 \mathrm{~h}$ sur $24 \mathrm{~h}$, il $\mathrm{y}$ a toujours un opérateur prêsent. Le planning ainsi défini assure donc la présence constante d'un ouvrier et 
d'un seul sur un certain poste de travail.

Disons tout de suite que les journées normales, notées $\mathrm{J}$, ont pour raison essentielle d'assurer que les opérateurs travaillent en "noyenne" un certain nombre d'heures par semaine, qui, dans le cas présenté plus haut, est de 40 heures. Pendant les quarts de J, les opérateurs sont généralement affectés à d'autres tâches (maintenance, technique, ...) que celles requises par le poste où ils sont affectés. Lorsqu'il n'y a pas de "réserves" d'ouvriers, les ouvriers en J sont souvent les premiers appelés pour remplacer les absents.

Dans le planning précédent, les 5 ouvriers sont attachés exclusivement à un seul et même poste de travail réclamant une présence permanentede $24 \mathrm{~h}$ sur $24 \mathrm{~h}$. Nous parlons alors de monovalence. Dans ce cas, il est bien évident que le nombre d'ouvriers affectés à un même poste est forcément un nombre entier. Cette hypothèse formait le cadre de travail de l'étude présentée dans (1).

Dans l'étude présente, nous rejetons cette hypothèse et considérons le cas général où à un poste de travail peut être affecté un nombre fractionnaire d'ouvriers. Pour éclairer les choses, nous donnons ci-après un planning cyclique sur 9 semaines.

Dans ce planning, les opérateurs 1, 2, 3, 4 sont affectés exclusivement à un même poste - disons le poste 1 -, et les opérateurs $6,7,8,9$ sont affectés exclusivement à un deuxième poste - le poste 2 -. L'opérateur 5 a un rôle particulier et est communément appelé "briseur de quarts". On remarquera, en effet, qu'il passe alternativement d'un poste sur un autre et que ceci permet d'assurer qu'il y ait chaque "jour" un $N$, un $M$, et un $S$ sur chacun des 2 postes, autrement dit, chacun des 2 postes est bien sous contrôle continu.

Nous parlerons de multibriseurs et de multivalence (1) dans le cas général où il n'y a pas forcément qu'un seul ouvrier capable de passer d'un poste sur un autre. Pour que cette hypothèse soit recevable, il est bien évident qu'il faut trouver au moins un ouvrier capable de "tenir" 2 ou plus de 2 postes différents. Ceci est en fait

(1) terme souvent confondu avec celui de polyvalence, qui signifie en fait tout autre chose. 


\begin{tabular}{|c|c|c|c|c|c|}
\hline Semaine & 1 & 2 & 3 & 4 & 5 \\
\hline Opérateurs & I M M JV. . & $I M M J V$. & I M M J V.. & $L$ M M J V.. & I M M J V .. \\
\hline 1 & $N N \cap N N-$ & $\mathrm{J} J \mathrm{~J} J \mathrm{~J}$ & $-M M M M M$ & $M-S S S S S$ & $S S-N N N N$ \\
\hline 2 & $S S S S-N N$ & $\mathrm{NNNNN}$ & J J J J J - - & $-M M M M M$ & $M-S S S S S$ \\
\hline 3 & $M M M-S S S$ & $S S S S-N N$ & $\mathbb{N} N \mathbb{N} N$ & $J J J J J$ & $-\mathrm{MMMMMM}$ \\
\hline 4 & $--M M M M$ & $M M M-S S S$ & $S S S S-N \mathbb{N}$ & $N N N N N-$ & $\mathrm{J} J \mathrm{~J} J \mathrm{~J}-{ }_{-}$ \\
\hline $5=$ bivalent & $\mathrm{NNN}-\cdots$ & $\ldots-\ldots M M$ & $M M M-S S S$ & $S S S S-N N$ & N N N N N - - \\
\hline 6 & $S S-\mathbb{N} U N N$ & $N N N \cdots$ & $-\ldots M M M$ & $M M M-S S S$ & $S S S S-N N$ \\
\hline 7 & $M-S S S S S$ & $S S-N N N N$ & $\mathrm{NNN}-\cdots$ & $--M M M M$ & M M M - S S S \\
\hline 8 & $-M M M M M M$ & $M-S S S S S$ & $S S-N N N N$ & $\| N N N \cdots$ & $---M M M M$ \\
\hline 9 & $J J J J J-$ & $-M M M M M$ & $M-S$ S S S S & $S S-N N N$ & $\mathrm{NN} \mathrm{N}_{-}--$ \\
\hline
\end{tabular}

\begin{tabular}{|c|c|c|c|c|}
\hline Semaine & 6 & 7 & 8 & 9 \\
\hline Opérateurs & L M M JV.. & I M M JV.. & L M M J V.. & I M M J V . \\
\hline 1 & $\mathbb{N N N}_{N}-\cdots$ & $---M M M$ & $M M M-S S S$ & ISSSS-NN \\
\hline 2 & $S S-N N N N$ & $\mathrm{NNN}-\cdots$ & -- M M M M & $M M M-S S S$ \\
\hline 3 & $M-S S S S S$ & $S S-N N N N$ & $\mathrm{NNNN} \cdots-$ & $--M M M M$ \\
\hline 4 & $-M M M M M$ & $M-S$ S S S S & $S S-N N N N$ & $\mathrm{~N} \mathrm{~N} \mathrm{~N}-\cdots$ \\
\hline $5=$ bivalent & $J J J J J-$ & $-M M M M M$ & $M-S S S S S$ & $j S-N N N N$ \\
\hline 6 & $N N N \mathrm{~N} N-$ & J J J J J - - & $-M M M M M$ & $M-S S S S S$ \\
\hline 7 & $S S S S-N N$ & $\mathrm{NNNNN}-$ & J J J J J - - & $-M M M M M M$ \\
\hline 8 & $M M M-S S S$ & $S S S S-N N$ & $\mathrm{~N} \mathrm{~N} \mathrm{~N} \mathrm{~N} \mathrm{N-} \mathrm{-}$ & $\mathrm{J} J \mathrm{~J} J \mathrm{~J}-$ \\
\hline 9 & $---M M M$ & $M M-S S S$ & $S S S S-N N$ & $\mathbb{N N N N N -}$ \\
\hline
\end{tabular}

TABLEAU 2 - Roulements de quarts en multivalence sur 2 postes, un ouvrier bivalent (pratiqué dans une usine de chimie de la région marseillaise). 
possible plus fréquemment qu'on ne pense, soit que les postes ne demandent pas de qualifications particulières, soit que les qualifications soient très proches.

Avant d'entamer l'étude, disons qu'il est très fréquent de trouver dans l'industrie et dans les services des plannings dans. lesquels 2 postes sont assurés en liaison avec un ou plusieurs briseurs de quarts. Le cas le plus fréquent est celui où l'on rencontre des plannings du type du Tableau 2.

Notre étude a pour but de donner les moyens de construire automatiquement des plannings de roulements de quarts dans lesquels puissent figurer des "multibriseurs".

On aura ainsi la possibilité d'assurer par exemple :

2 postes avec $9,10,11,12 \ldots$ ouvriers

3 postes avec $12,13,14,15, \ldots$ ouvriers

........

Pour ne pas reprendre les diverses considérations présentées dans (1) sur les journées normales, les passages de consigne, les weekends, etc..., nous restreindrons notre étude à la définition des cycles "bruts" c'est-à-dire ceux où l'on se désintéresse du positionnement des journées normales.

\section{DEFINITION DU PROBLEME}

Pour faciliter le problème, nous le présenterons dans une version légèrement simplifiêe.

Etant donné

- un nombre $P$ d'ouvriers,

- un nombre $R$ de postes à assurerde façon continue par les $\mathrm{P}$ ouvriers,

- un nombre minimum de jours de repos devant succêder à chacun des tours (N, S, M),

- des bornes inférieures a et supérieures b pour la longueur (i.e. le nombre de quarts) de chacun des tours ( $N, S$ ou $M)$, 
il s'agit de trouver un moyen d'engendrer tous les plannings cycliques vérifiant ces hypothèses et tels que chaque ouvrier ait, à un décalage près dans le temps, exactement le même cycle que les autres ouvriers.

Nous supposerons, d'autre part, que les tours d'un cycle doivent se succéder dans un ordre détermine qui peut être le suivant : un tour de nuits, puis un tour de soirs, puis un tour de matins, puis de nouveau un de nuits, etc...

\section{RESULTATS GENERAUX}

Nous donnons un premier résultat dont la dểmonstration est triviale en considérant que chaque "jour" il y a un nombre constant de $\mathrm{N}$, de $\mathrm{S}$ et de $\mathrm{M}$.

Lemme 1 : Considérons le planning commun à $P$ ouvriers et concernant $R$ postes. Si un ouvrier termine un tour (de $\mathrm{N}$ par exemple) à une date $t$ (le dernier quart du tour se trouve à $t$ ), il y a alors nécessairement un autre ouvrier qui commence un tour de $\mathrm{N}$ à la date $t+1$.

ce lemme nous sera utile pour définir un algorithme permettant d'engendrer le cycle.

Nous aurons aussi besoin du résultat suivant.

Lemme 2 : Avec $\mathrm{P}$ ouvriers assurant $\mathrm{R}$ postes, le nombre total $\mathrm{n}$ de $\mathrm{N}$ (ou de $S$, ou de $M$ ) dans le cycle est :

$$
n=\frac{L R}{P}
$$

ou I est la longueur d'un cycle. Le nombre $n$ doit être un multiple entier de 7 .

La longueur $L$ des cycles vérifie alors

$$
\mathrm{I}=\mathrm{n} \frac{\mathrm{P}}{\mathrm{R}}
$$

et I doit être entier et multiple de 7 .

\section{Démonstration:}

Chaque "jour" on doit trouver $R$ nuits d'où au total LR nuits dans le planning commun au $\mathrm{P}$ ouvriers. Comme les ouvriers ont tous des mêmes cycles, on doit donc trouver $L R / P$ nuits (ou soirs, ou matins) dans un cycle. D'autre part, du fait de "l'égalité" des cycles, chaque ouvrier doit faire un même 
nombre de nuits (ou de soirs,...) pour chaque jour de la semaine. On a donc $n=L R / P$ et $n$ doit être un multiple de 7 . on en déduit immédiatement les propriétés de $I$.

\section{THEOREME CONCERNANT LES PLANNINGS EN DECALAGES REGULIERS}

Dans (1) nous avons donné une méthode gênêrale permettant d'obtenir tous les cycles possibles en monovalence. Nous avons vu, d'autre part, que la connaissance des débuts de cycles dans un planning cyclique permettait d'améliorer sensiblement la rapidité de définition des cycles. Dans ce qui suit, nous nous baserons à nouveau sur l'hypothèse que l'on recherche des cycles tels qu'ils puissent être rangés en décalage régulier dans un planning.

\section{Précisons de quoi il s'agit :}

Un planning cyclique (c'est-à-dire se reproduisant identiquement à lui-même) est dit en décalage rêgulier si les cycles de chacun des $\mathrm{P}$ opérateurs débutent à des dates régulièrement espacées. Comme il s'agit de problèmes cycliques, le mot "débuter" n'a, en fait, guère de signification. Nous l'utiliserons cependant dans le sens suivant : par convention, le cycle du premier opérateur (en haut du planning) débutera un lundi et il commencera avec le plus petit tour de nuits. (En toute généralité, ce cycle pourra effectivement débuter un autre jour de la semaine, il suffit pour cela de le décaler de 1, 2, ... 7 jours). Deux opérateurs seront dits en décalage de $d$, si le planning du premier s'identifie, après un aécalage de d jours (modulo la longueur du cycle) avec le planning du second opérateur. Nous dirons qu'un planning cyclique pour $P$ ouvriers est en décalage régulier s'il est possible de réordonner les lignes du planning de façon que les cycles apparaissent, en descendant, avec un décalage constant et dans le même sens.

Avant de donner un théorème fondamental sur la non redondance des plannings cycliques, nous allons démontrer quelques résultats préliminaires. 
Lemme 3 : Solt un planning $(P, R)$ definissant le planning de $P$ ouvriers assurant $R$ postes. Si dans ce planning les cycles de chacun des opëratenrs $i=1, \ldots, p$ débutent à des dates $t_{i}$ régulièrement espacêes de d :

$$
t_{i}=I+(i-1) d, i=I, \ldots, P
$$

alors le planning $(P, R)$ considêrê de la date $t=1$ a la date $t=P d$ se reproduit identiquement à luimême.

\section{Dëmonstration:}

En supposant que 1 'ouvrier 1 (haut du planning) fasse un $N$ à la date $t=1$ (ce que l'on peut toujours supposer nous allons tout d'abord démontrer qu'il fait nécessairement un $\mathrm{N}$ à la date $t=P d+1$. comme chaque jour il y a $R$ "N" à assurer, nous allons supposer que ces $R$ "N" sont effectués à la date $t=P d+1$ par des ouvriers $i_{1}, i_{2}, \ldots i_{R^{\prime}}$ avec $i_{j} \neq 1, \forall_{j}=1, \ldots R$. Du fait de l'égalité des cycles, $i l$ faut alors introduire $R$ " $N$ " à la date $t=1+(P-1)$ a pour les opérateurs $i_{j}-1$, $j=1, \ldots$, R. Par ailleurs l'ouvrier $P$ fait déjà une "N" $\mathrm{a}$ la date $t=1+(P-1)$ d et puisque $i_{j}-1 \neq P, y_{j}=1, \ldots, R$ on trouverait donc $R+1$ " $N "$ en $t=1+(P-1) d$. Comme ceci est impossible, c'est que I'ouvrier 1 reprend bien une " $N$ " en $t=P d+1$. De proche en proche on démontre alors trivialement le résultat.

Pour faciliter les choses, nous allons nous intéresser aux plannings $(P, R)$ dont la longueur $I$ est un multiple de $P d^{(1)}$ (d multiple de 7). Dans l'intervalle de temps défini par $L$ on peut définir en gênéral de nombreux plannings en décalage régulier de d avec Pd diviseur de $L$.

(1) Si $P$ est divisible par $R$ il $y$ a en fait de nombreux plannings possibles de longueur $L$ inférieure à un multiple de Pd, mais ces plannings se retrouvent dans ceux dont la longueur est un multiple de Pa. 
Nous allons voir que certains de ces plannings peuvent être redondants par rapport à d'autres. Pour préciser le vocabulaire, soient 2 plannings de longueur $\mathrm{L}$ en décalages réguliers de $d_{1}$ et $d_{2}$ respectivement. Nous dirons que les plannings $\left(\bar{\alpha}_{2}\right)$ sont redondants par rapport aux plannings $\left(d_{1}\right)$ si toute configuration que l'on peut obtenir en imposant que les débuts de cycles soient en décalage de $d_{2}$ peut être obtenue en imposant que les débuts de cycles soient en décalage de $d_{1}$.

THEOREME : Soient deux familles de plannings en décalages réguliers, l'une en décalage de $d_{1}$ pour $P$ opérateurs, I'autre en décalage de $\mathrm{d}_{2}$ pour $\mathrm{P}$ opérateurs. Supposons que $\mathrm{Pd}_{1}$ et $\mathrm{Pd}_{2}$ soient des diviseurs d'un même nombre $I$. Une condition nécessaire est suffisante pour que les plannings engendrés par $a_{2},\left(d_{2}<a_{1}\right)$ soient redondants par rapport aux plannings engendrés par $d_{1}$ est que

(1) $P d_{1}$ divise $\left(\mathrm{d}_{1}, \mathrm{Pd}_{2}\right)^{+}$

et

(2) $\mathrm{Pd}_{2}$ divise $\left(\mathrm{Pd}_{1}, \mathrm{~d}_{2}\right)^{\text {* }}$

où l'on note $(a, b){ }^{*}$ le p.p.c.m de $a$ et $b$.

Démonstration :

soient $n_{1}$ et $n_{2}$ tels que

$\mathrm{n}_{1} \mathrm{Pd}_{1}=\mathrm{n}_{2} \mathrm{Pd}_{2}=\mathrm{L}$

et supposons $\mathrm{d}_{2}<\mathrm{d}_{1}$

D'après le lemme 3 on a les considérations suivantes :

Dans un planning $d_{1}$ on a des nuits aux dates (que l'on peut considérer comme des lundis) :

$s_{i h}^{(1)}=1+(i-1) d_{1}+h P d_{1}$,

$i=1, \ldots$, p et $h=0, \ldots, n_{1}-1$. 
Dans un planning $\mathrm{d}_{2}$ on a des nuits aux dates

$$
\begin{aligned}
& s_{j k}^{(2)}=1+(j-I) a_{2}+k P d_{2} \\
& j=I, \ldots, P \text { et } k=0, \ldots, n_{2}-1
\end{aligned}
$$

Nous allons Etablir le théorème par une suite de résultats.

Résultat 1 : Nous allons voir tout d'abord que les deux suites (s ${ }^{(1)}$ ) et $\left(s_{j k}^{(2)}\right.$ ) ainsi définies ont nécessairement au moins 2 éléments en commun $(\mathrm{si} p>1)$. Comme $\mathrm{s}_{10}^{(1)}=\mathrm{s}_{10}^{(2)}$ il reste à trouver un deuxième élément.

Il est clair que les deux équations suivantes, en $i, h$, $j, k$ :

$$
\begin{aligned}
& (i-1)+h P=n_{1} \\
& (j-1)+k P=n_{2} \\
& (j-1, P), h \varepsilon(1, P) \quad k \in\left(0, n_{2}\right) .
\end{aligned}
$$

ont au moins une solution et que cette solution ne peut être ( $i=1, j=1, h=0, k=0$ ) puisque $d_{2}<a_{1}$, donc $\left.\mathrm{n}_{2}>\mathrm{n}_{1}\right)$. D'autre part cette solution vérifie

$$
h<n_{1} \text { et } k<n_{2} \quad(p>1)
$$

D'où l'existence de $i, h, j, k$ tels que

$$
\mathrm{s}_{i \mathrm{~h}}^{(1)}=1+\mathrm{n}_{1} \mathrm{~d}_{1}=\mathrm{s}_{\mathrm{jk}}^{(2)}=1+\mathrm{n}_{2} \mathrm{~d}_{2}=\frac{\mathrm{L}}{\mathrm{P}}<\mathrm{L} \quad(\mathrm{P}>1)
$$

Ce qui établit le résultat.

Résultat 2: Considérons maintenant l'ensemble des éléments communs aux deux suites finies $\left(s_{i h}^{(1)}\right)$ et $\left(s_{j k}^{(2)}\right)$.

Soit $\Omega=\left(t_{r}, r=1,2, \ldots\right)$ 
cet ensemble, et notons $\left(i_{r}, h_{r}\right)\left(j_{r}, k_{r}\right)$ les êléments correspondants à $t_{r}$. D'après le résultat 1 cet ensemble contient au moins 2 éléments.

Nous allons examiner deux propriétés possibles de $\Omega$ :

\section{Propriété 1 :}

sif $\left(t_{r}, t_{s}\right), r \neq s$ tels que l'on ait $\left(i_{r}=i_{s}\right.$ et

$j_{r} \neq j_{s}$ ) alors les décalages $d_{1}$ et $d_{1}$ ne peuvent engendrer

les mêmes plannings : en effet, dans ce cas, un au moins

des opérateurs d'un planning $d_{1}\left(\mathrm{ou} d_{2}\right.$ ) a au moins 2 "N"

à faire tandis que dans les plannings $d_{2}$ (ou $d_{1}$ ) ces 2 "N" sont répartis entre 2 opérateurs différents.

\section{Propriété 2 :}

$$
\begin{aligned}
& \text { si } \forall\left(t_{r^{\prime}} t_{S}\right), r \neq s \text { on } a \\
& \left(i_{r}=i_{s} \text { et } j_{r}=j_{S}\right) \quad(\operatorname{cas} a)
\end{aligned}
$$

ou bien

$\left(i_{r} \neq i_{s}\right.$ et $\left.j_{r} \neq j_{s}\right) \quad($ cas $b)$

alors la famille des plannings engendrés par $d_{2}$ est une sous famille de celle des plannings en décalage de $d_{1}$.

Pour établir cette proprété, nous allons voir qu'il est impossible d'avoir le cas (a) uniquement. Supposons en effet.

$$
\left(i_{r}=i_{s} \text { et } j_{r}=j_{s}\right), \forall\left(t_{r}, t_{s}\right), r \neq s \text {. }
$$

On a alors : $t_{r}-t_{s}=$ multiple de $\mathrm{Pd}_{1}$

$$
\text { = multiple de } \mathrm{Pd}_{2}
$$

avec $t_{r}-t_{s}<L$. Or, d'après le Lemme 3 , les plannings de longueur $L$ ainsi définis ne peuvent être que des répétitions des sous-plannings définis avec les mêmes $d_{1}$ et $d_{2}$ sur une longueur $I^{*}=\left(P d_{1}, P d_{2}\right)^{*}$. 
Autrement dit on aurait $\mathrm{Pd}_{1}$ et $\mathrm{Pd}_{2}$ divise $t_{r}-t_{S}<\mathrm{L}^{\text {q }}$, ce qui est impossible.

Raisonnons alors sur le cas (b) qui est alors certain si l'on $n^{i}$ a pas les conditions de la proprieté 1.

$$
\text { Soient: } t_{1}, t_{2}, \ldots t_{p}
$$

les dates ol $1^{\prime}$ on retrouve les dëbuts de cycles dans les 2 plannings $\left(a_{1}\right)$ et $\left(a_{2}\right)$. Examinons tout $a^{\prime}$ abord 1 'exemple suivant portant sur $p=5, d_{1}=2, a_{2}=1$.

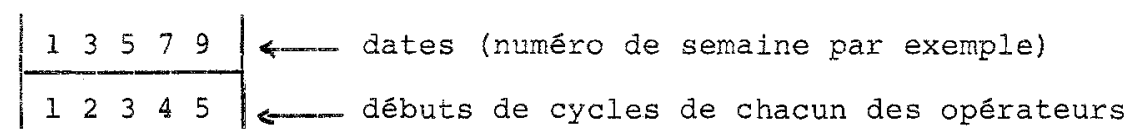
$\mid \begin{array}{llll}13 & 5 & 79 \\ 1234512345 & \text { dates (numéro de semaine par exemple) }\end{array}$ On a :

\begin{tabular}{|cccccc|}
$r$ & 1 & 2 & 3 & 4 & 5 \\
\hline$t_{r}$ & 1 & 3 & 5 & 7 & 9 \\
\hline$t_{r}$ & 1 & 2 & 3 & 4 & 5 \\
$j_{r}$ & 1 & 3 & 5 & 2 & 4 \\
\hline
\end{tabular}

On est bien dans le cas (b). Définissons alors :

$$
j_{r}^{\prime}=i_{r}, r=1,2, \ldots
$$

On $a: j_{1}^{\prime}=1, j^{\prime}{ }_{2}=2, j^{\prime}{ }_{3}=3, j{ }_{4}=4, j^{\prime}{ }_{5}=5$.

In renumérotant les opérateuxs de $d_{2}$ on arrive ainsi à voir que les plannings $d_{2}$ peuvent être obtenus avec les plannings $d_{1}$. Pour que ce ne soit pas possible, il faudrait que pour $2 j_{r}: j_{r_{1}}$ et $j_{r_{2}}$ avec $j_{r_{1}}=j_{r_{2}}$ on ait $i_{r_{1}} \neq i_{r_{2}}$. Or ceci contredit le cas (b). 
Il nous reste à montrer que la condition

(0) $]\left(t_{r}, t_{s}\right)$ :

$$
\left(i_{r}=i_{s} \text { et } j_{r} \neq j_{s}\right) \quad(\operatorname{cas} 0-1)
$$

$$
\left(i_{r} \neq i_{s} \text { et } j_{r}=j_{s}\right) \quad(\operatorname{cas} 0-2)
$$

est équivalente à la suivante :

(1)

$$
\mathrm{Pd}_{1} \text { ne divise pas }\left(\mathrm{d}_{1}, \mathrm{Pd}_{2}\right)^{*} \quad \text { (cas 1-1) }
$$

$$
\left.\mathrm{Pd}_{2} \text { ne divise pas }\left(\mathrm{Pd}_{1}, \mathrm{~d}_{2}\right)^{+} \quad \text { (cas } 1-2\right)
$$

Plaçons nous dans le cas $(0-1)$

Posons $D=t_{s}-t_{r}$. On a alors

$$
\begin{aligned}
D & =\text { divisible par } P d_{1} \\
& =\text { non divisible par } P d_{2} \\
& =\text { multiple de } d_{2}
\end{aligned}
$$

autrement dit, $P d_{2}$ ne divise pas un multiple de $\left(P d_{1}, d_{2}\right)^{*}$ donc ne divise pas $\left(P d_{1}, d_{2}\right)$.

La réciproque est immédiate en partant d'un multiple D de $\mathrm{Pd}_{1}$ vêrifiant les propriétés prêcédentes.

L'examen du cas $0-2$ se fait parallèlement.

Le théorème prêcédent nous donne ainsi un moyen de se fixer des décalages réguliers non redondants avec d'autre.

\section{ALGORITHME DE GENERATION DE PLANNINGS EN MULTIVALENCE}

Nous admettrons dans ce qui suit que les plannings communs aux $P$ ouvriers assurant $R$ postes sont constitués de cycles en décalages réguliers.

Nous savons que chaque ouvrier doit assurer $n$ "N" dans son cycle, $n$ "S" et $n$ "M". 
Dans un planning cyclique de longueur $I$ on doit trouver $R$ "N" (ou "S" ou "M) chaque jour.

Occupons nous $d^{y}$ abord de remplir le planning en assurant un " $N$ " chaque jour. Du fait du Lemme 3, nous supposons $L=$ Pd. Pour assurer un "N" chaque jour, il faut alors porter $\mathrm{A}$ "N" dans le cycle commun aux $P$ opérateurs. Comme les tours de $N$ ne peuvent excéder certaines longueurs, nous effectuons un découpage de $d$ en $d_{1}, d_{2}, \ldots$ $d_{1}+d_{2}+\ldots=d$ et $d_{i}$ est la longueur $d$ 'un "ième" tour de nuits. En tenant compte des débuts de cycles on opère alors comme dans (1) pour le positionnement de ces $d$ " $N$ ". On opère de même pour assurer un "S" et un " $M$ " chaque jour. Si $R=1$ on a obtenu ainsi un planning. Si R = 2 il nous faut positionner une seconde batterie de d "N", de d "s" et de d "M". (Nous parlons alors de nuit-bis, de soir-bis,...) et ceci en tenant toujours compte des débuts de cycles et des diverses contraintes, en particulier de celles concernant les jours de repos entre les tours. On continue jusqu'à ce que chaque jour on ait $R$ " $N$ ", $R$ "S" et $R$ " $M$ " dans le planning.

Nous donnons ci-après deux exemples obtenus par ordinateur :

Exemple 1 : tours de 3 et 4 quarts, 9 ouvriers, cycle de 9 semaines $-N, A=$ nuits $;, B=$ soirs $;, C=$ matins

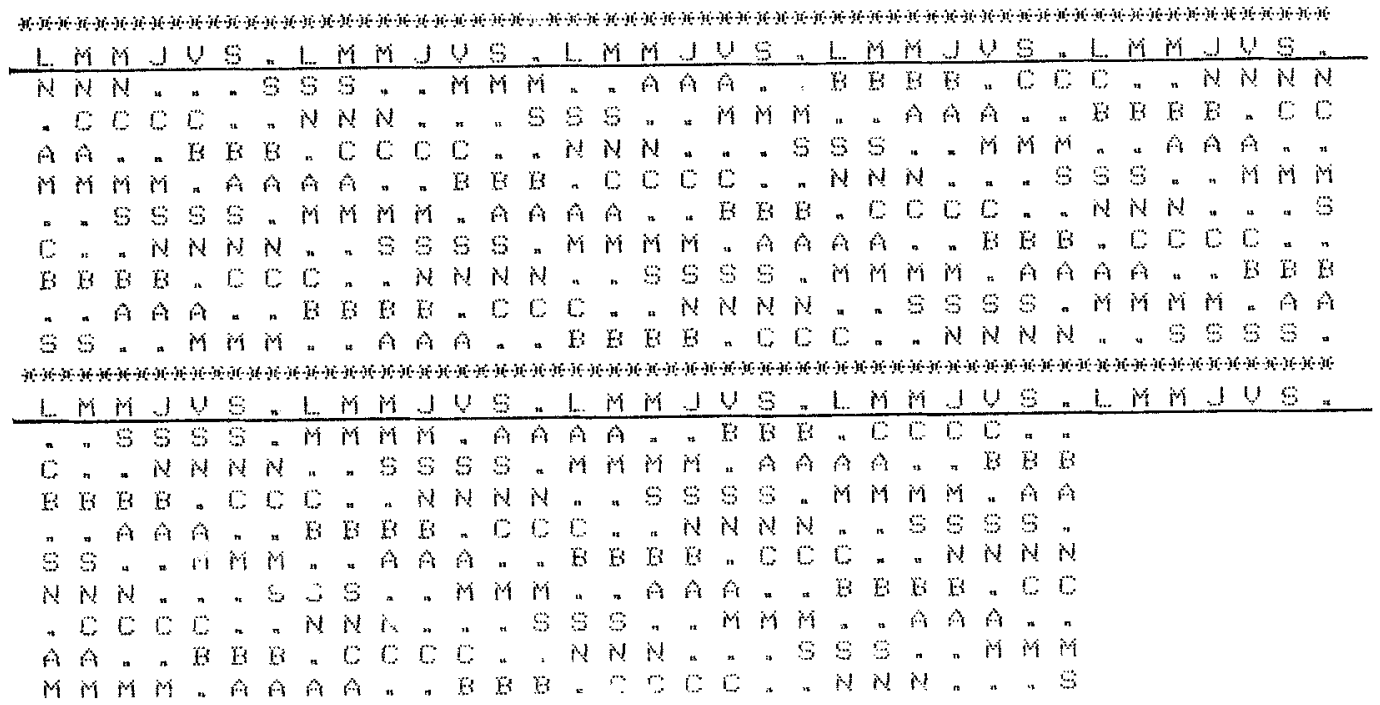


Exemple 2 : tours de 5 quarts, 9 ouvriers, cycle de

45 semaines :

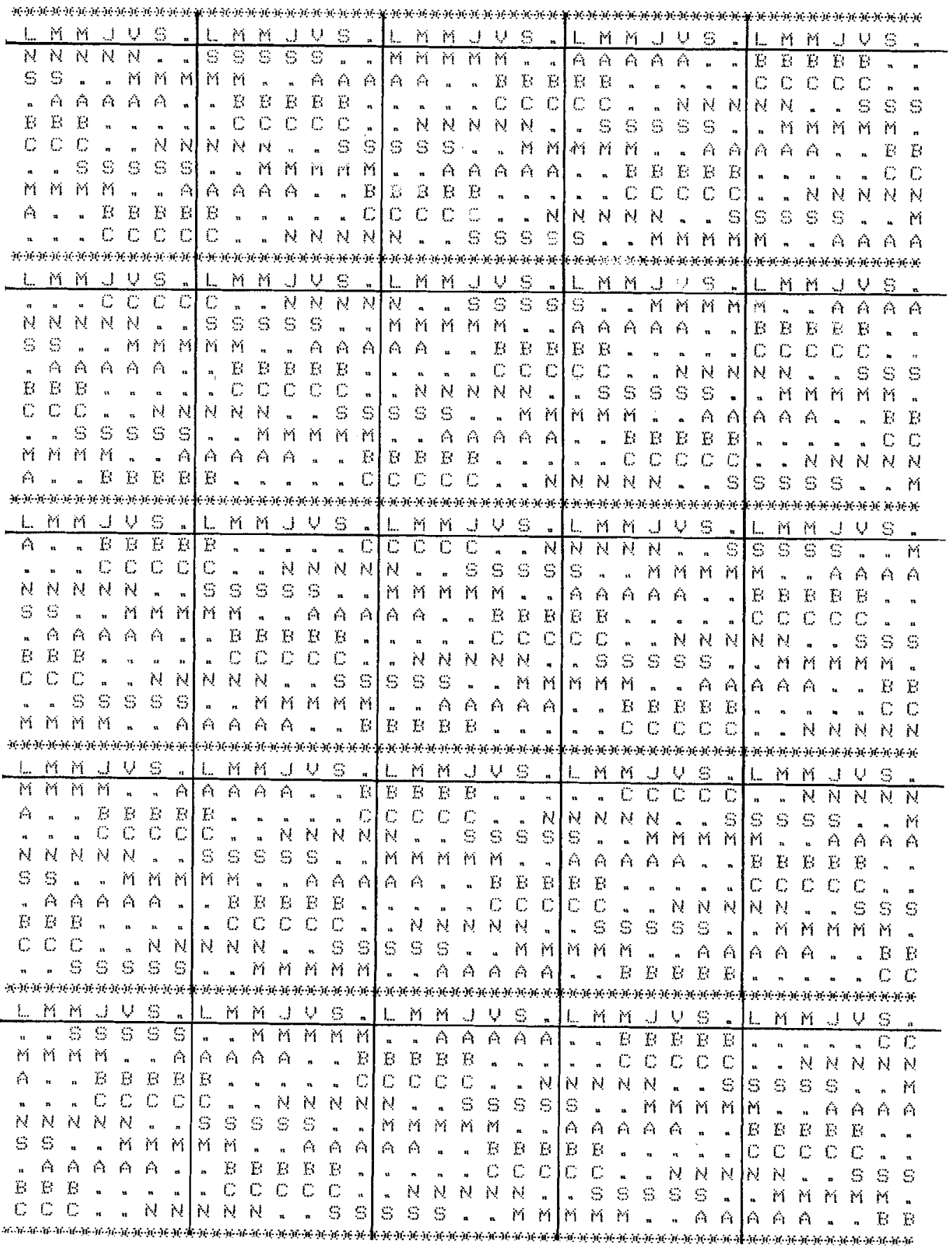


Exemple 2 (suite)

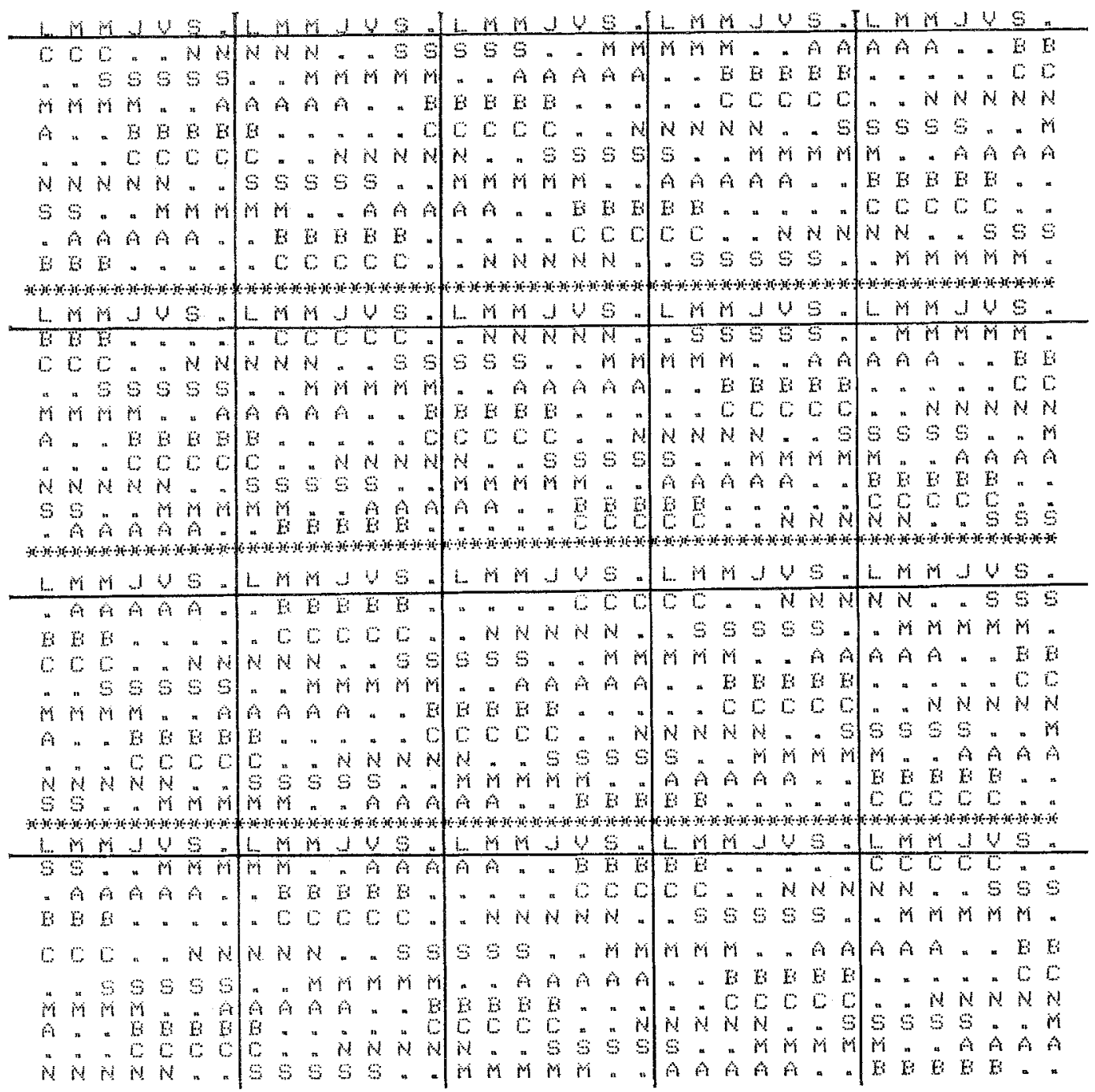




\section{LA RECHERCHE DES BRISEURS DE QUARTS}

Le planning étant ainsi obtenu, il reste à répartir les ouvriers suivant les postes. Soient $\mathrm{P}_{1}, \mathrm{P}_{2}, \ldots, \mathrm{P}_{\mathrm{R}}$ les $\mathrm{R}$ postes.

Une manière d'opérer est de construire une arborescence dont on comprendra facilement le principe à partir de l'exemple suivant où il s'agit de répartir les ouvriers entre 2 postes :

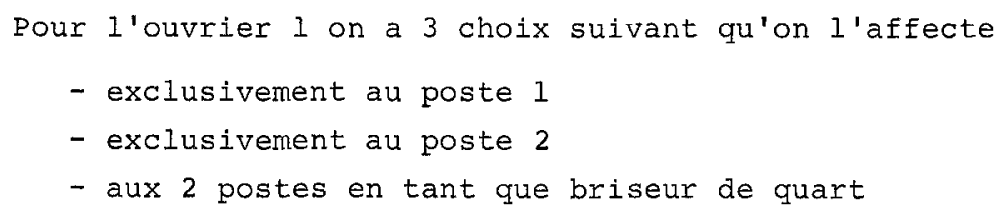
bre de possibilités est bien plus considérable). En se référant au planning on peut éliminer certaines sous-arborescences issues de certains arcs.

Par exemple, une sous-arborescence pour laquelle la racine est extrémité d'un chemin pour lequel on trouverait par exemple deux ouvriers sur un même poste effectuant le même jour un même quart, est à supprimer.

Les chemins qui restent et qui vont de la racine aux noeuds terminaux fournissent des solutions possibles.

Nous donnons ci-après un exemple de planning obtenu par ordinateur avec un briseur unique $(1=$ poste $1,2 .=$ poste 2 , $B Q=$ briseur de quart). 


\section{Exemple 3}

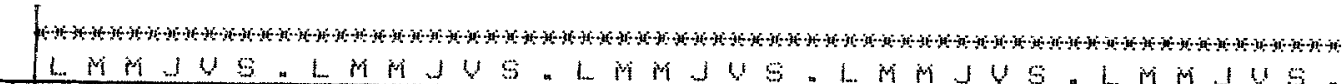

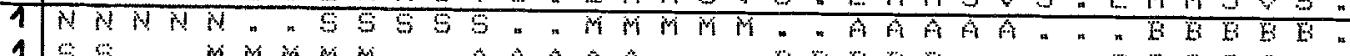

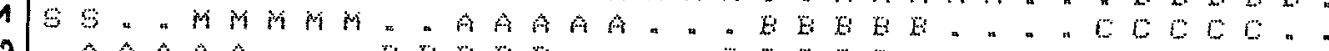

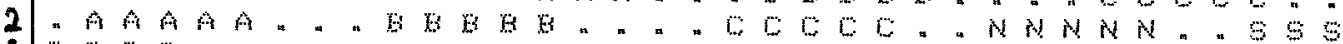

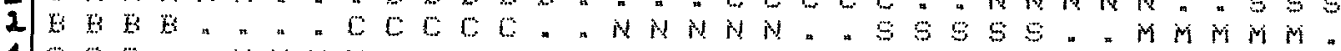

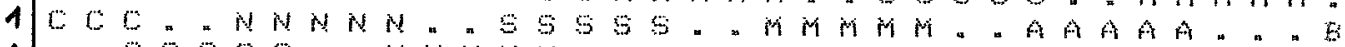

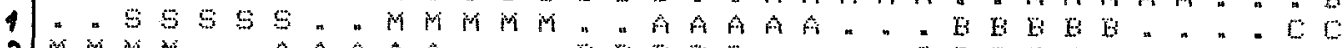

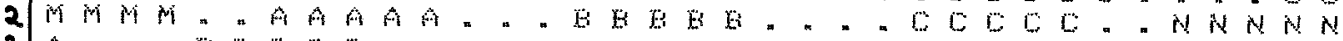

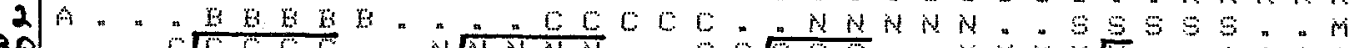

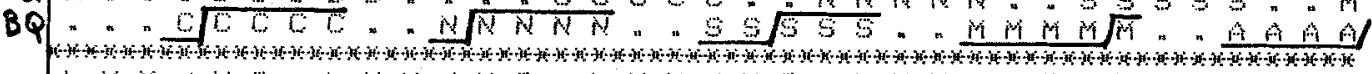

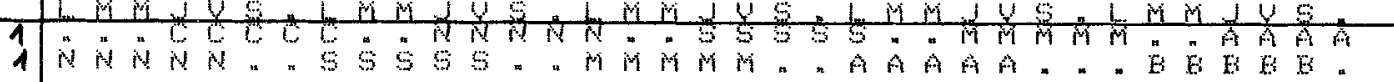

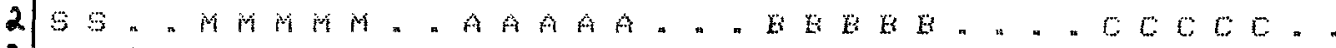

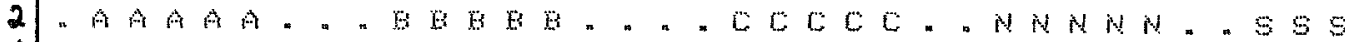

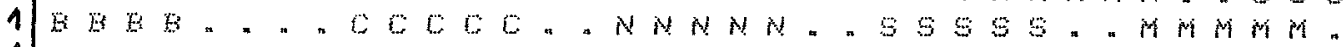

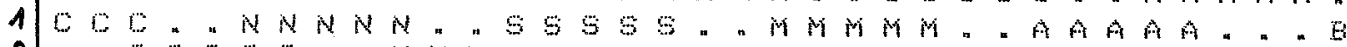

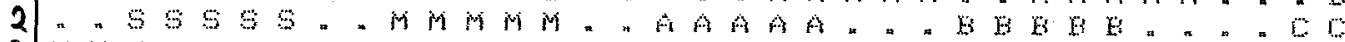

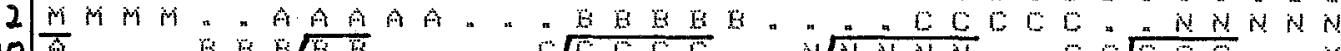

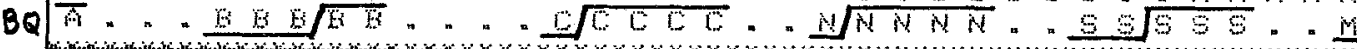
(x)

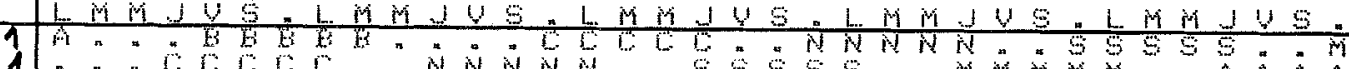

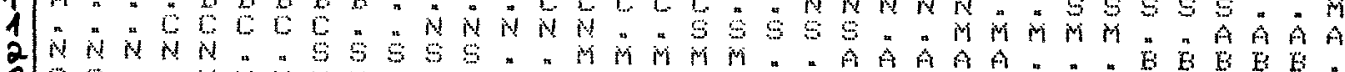

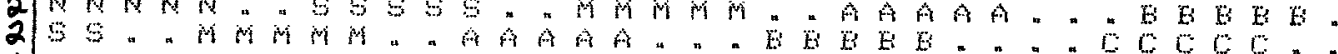

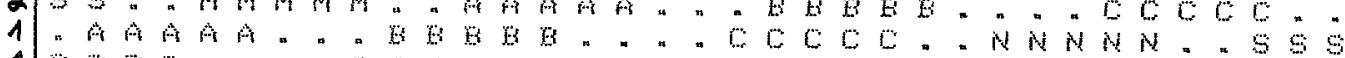

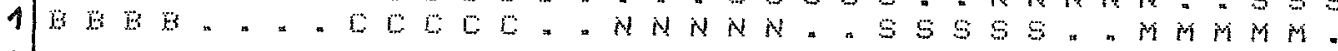

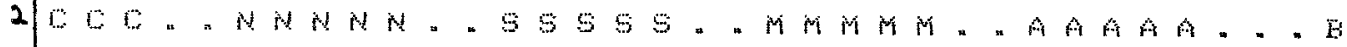

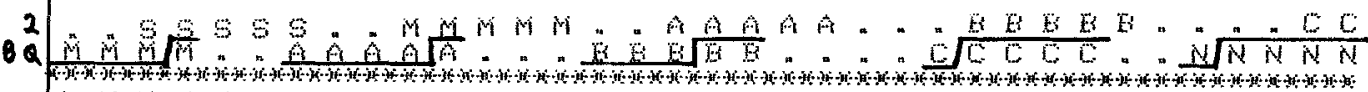

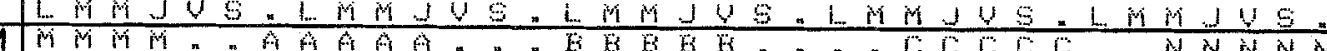

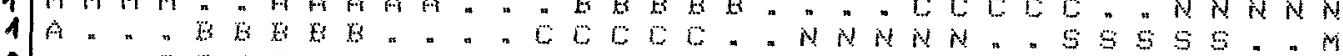

2."

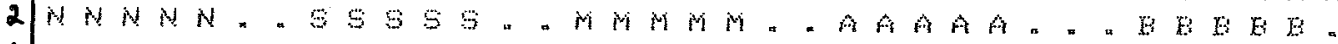

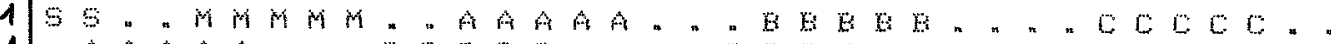

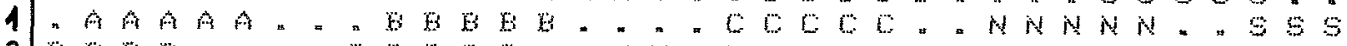

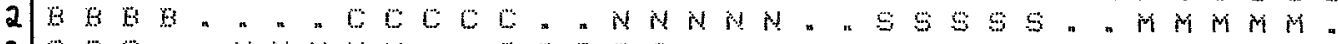

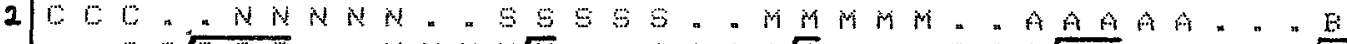

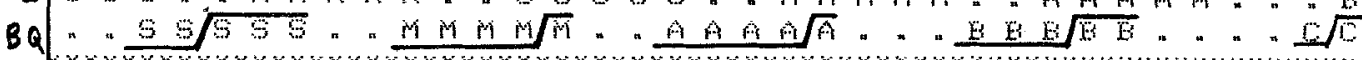

Thond

1 .

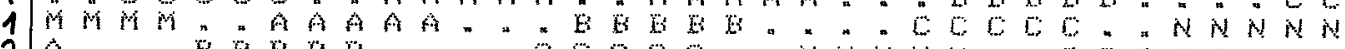

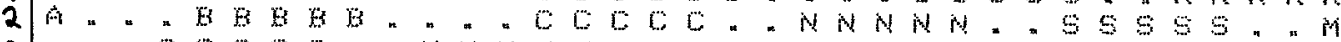

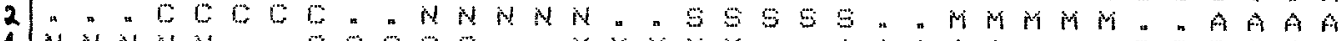

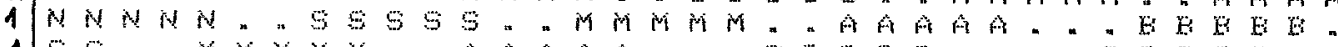

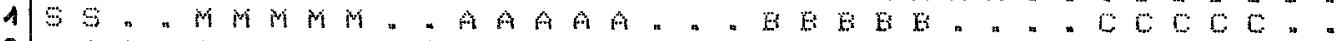

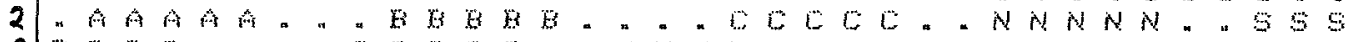

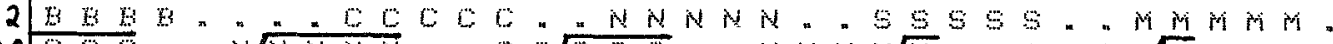

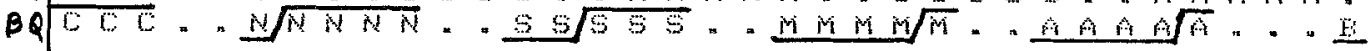


Fxemple 3 (suite)

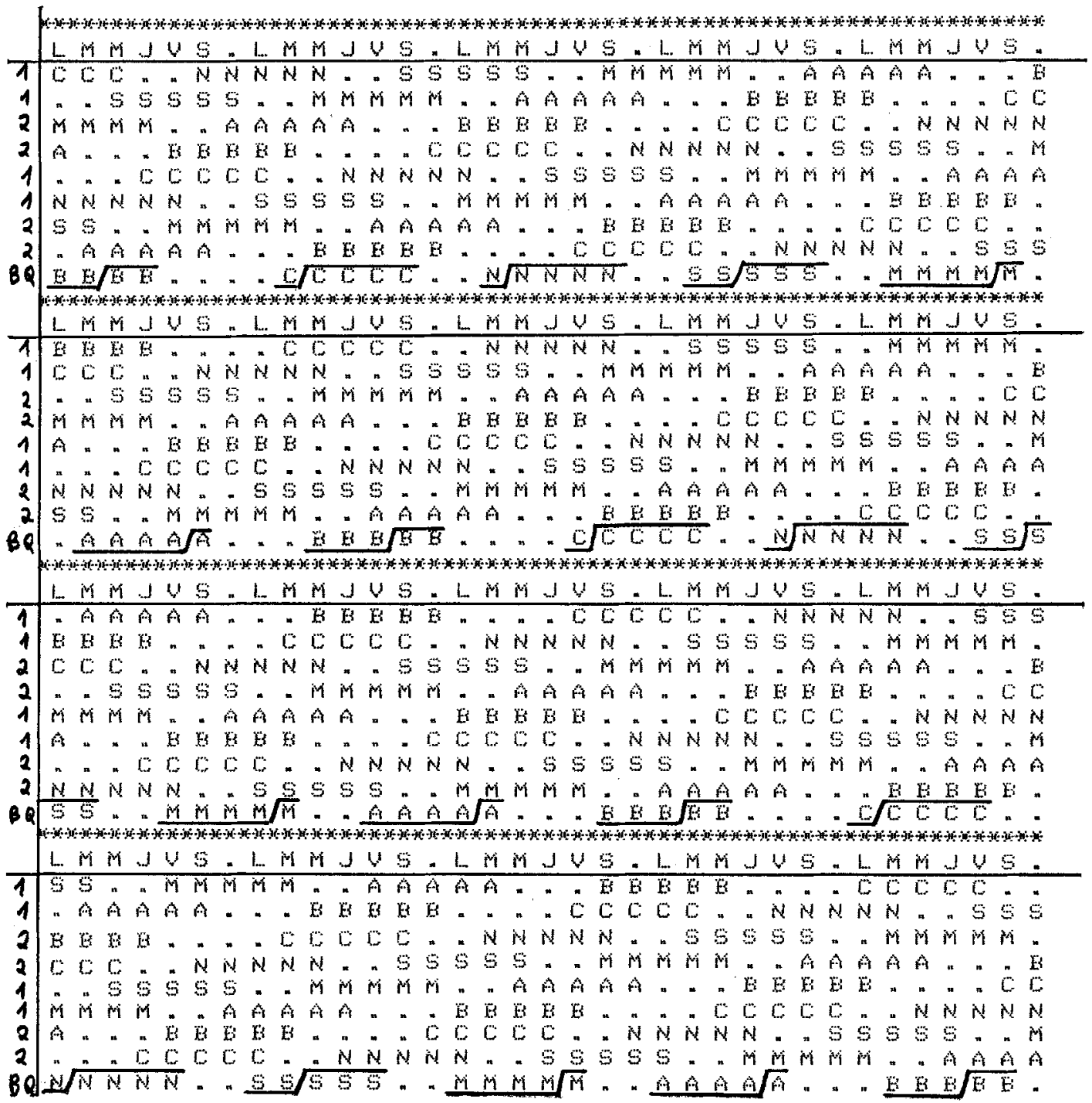




\section{REMARQUES}

on remarquera que la méthodologie présentée ici s'applique à des problèmes beaucoup plus généraux que celui où la même charge de travail doit être assurée $24 \mathrm{~h}$ sur $24 \mathrm{~h}$.

Par exemple, si l'on ne positionne, au moyen de l'algorithme, que des $N$, des $S$, des $M$, et, par exemple, des $M$ "bis", on aura dans le planning, chaque jour un $\mathrm{N}$, un $\mathrm{S}$ et deux $\mathrm{M}$; autrement dit, 2 ouvriers le matin au lieu de 1 le reste du temps sur un poste.

On peut, bien sûr, supprimer aussi dans les plannings obtenus un ou plusieurs quarts certains jours (par exemple le dimanche). La méthode permet ainsi d'engendrer tous les plannings cycliques en continu ou en semi-continu dans lesquels les ouvriers ont, à un décalage près dans le temps, les mêmes plannings de travail.

\section{Réfêrence}

1 - R. TREMOLIERES : Le problème des roulements de quarts pour les entreprises à feu continu.

(à paraître dans RAIRO, Janvier 1976)。 